\title{
Theologische Grundlegungen für eine Ethik der Social Media
}

\section{Einleitung}

Peter Arbeitsloser und Mildred Bürokraft haben sich noch im „echten Leben“" kennengelernt (vgl. Kling 2017, 30). Eines Tages beschlossen die beiden Figuren aus Marc-Uwe Klings Qualityland, ihre Profile bei Quality Partner abzugleichen. Kling $(2017,30)$ schreibt:

„Das System sagte ihnen, dass sie nicht zusammenpassen. Es präsentierte sogar jedem einen besseren Partner. Peter und Mildred haben sehr lange darüber nachgedacht und schließlich eingesehen, dass sie wirklich nicht zusammenpassen. Sich einfach mal, nur so zum Spaß, bei QualityPartner einzuloggen war gar nicht so richtig spaßig gewesen. Beide haben sich heimlich mit einem besseren Partner verabredet. [...] Peters beste Partnerin ist Sandra Admin. Sie streiten sich nie. Sandra sieht so gut aus, wie ein Mann auf Peters Level es sich nur erhoffen kann: mittel.“

Wollen wir so leben?1 Wollen wir so leben wie die Figuren in Marc-Uwe Klings satirischer Dystopie „QualityLand“, streitlos eingepasst, perfekt gematcht, von der Technik bestimmt? Angesichts scheinbar alternativloser Entwicklungen im digitalen Wandel, angesichts sich einschleifender Selbstverständlichkeiten - etwa der, für scheinbar kostenlose Angebote mit den eigenen Daten zu bezahlen, ohne nach dem Wert dieses Zahlungsmittels zu fragen ${ }^{2}$ - und gerade angesichts dessen, was Byung Chul Han seduktive „smarte Macht“ genannt hat, eine Macht, die nicht verbietet oder einschränkt, sondern mit besseren Lebensmöglichkeiten verführt (vgl. Han 2014, 25-7), wie QualityPartner, und gerade so das Leben verändert angesichts all dessen ist es die erste Aufgabe einer theologischen Ethik, diese Frage wachzuhalten: Wollen wir so leben? Wollen wir, dass die beeindruckenden Fortschritte in der Kommunikationstechnik auf diese Art und Weise genutzt werden? Oder geht es auch anders?

1 Die Frage ist angelehnt an Hans-Richard Reuters Formulierung der güterethischen Grundfrage „Wie wollen wir leben?““ (Reuter 2015, 114).

2 Vgl. dazu: Pariser 2012, 59. Den Hinweis auf die Wichtigkeit dieses Umstandes verdanke ich auch den Teilnehmer*innen meiner Übungen zur Digitalisierung und Digitalen Theologie, insbesondere Roland Hummel. 
Klings Dystopie, die manchmal erschreckend dicht an der gegenwärtigen Realität ist, evoziert diese Frage nach dem guten Leben. Auch bereits gegenwärtige Realitäten in digitalen Lebenswelten haben die ethische Reflexion des sozialen Netzes ${ }^{3}$ nötig gemacht. Dies und die notwendige thematische Breite der Reflexion zeigen schon die Aufsatztitel der Texte, die Peter Kemper, Alf Mentzer und Julika Tillmanns in einem Sammelband zur Wirklichkeit 2.0 zusammengestellt haben: „Das Ende der Privatsphäre“, „Virtuelle Nähe“, „Ausgekundschaftet“, „Cyberbullying“, „Digitales Selbst“, „Zur Faszination der Gewalt in Computerspielen“, „Macht und Autorität bei Wikipedia“ oder „Digitale Demokratie“ (Vgl. das Inhaltsverzeichnis in Kemper, Mentzer und Tillmanns 2012, 5-7). Wo soll eine Ethik, genauer: eine dezidiert theologische Ethik ansetzen, die diese sozialen Phänomene orientierend reflektiert?

\section{Ansatzpunkte: Für eine eschatologische Grundlegung}

Wo anfangen also? Diese Frage stellt sich mindestens zweimal. Zunächst bezieht sie sich auf die Sachmomente (Tödt 1988, 29), „Aspekte oder Elemente“ (Reuter 2015, 112) sittlicher Urteilsfindung, die die ethische Reflexion unterschieden hat. Hans-Richard Reuter (2015, 112-116) etwa differenziert vier Arbeitsschritte: die „Beschreibung des Kontextes“, die die Benennung empirischer Fakten, rechtlicher Regelungen, von Machtverhältnissen und Üblichkeiten beinhaltet, die "Identifizierung der ethischen Perspektive(n), Kriterien und Orientierungen", deren Prüfung und Abwägung und schließlich „Entscheidung und Umsetzung“. Während die Urteilsfindung mit Tödt (1988, 29; Hervorhebung im Original) als „iterativer Prozeß“ zu verstehen ist, in dem die unterschiedlichen Momente aufs engste zusammenhängen, muss die Reflexionsarbeit irgendwo anfangen - und dies prägt die weitere Reflexion: Wer bei der Situations- und Kontextbeschreibung anfängt, steht in der Gefahr, sich von empirischen Fakten, technischen (Un)Möglichkeiten oder der positiven Rechtslage auch den evaluativen Rahmen vorgeben zu lassen. Wer bei den ethischen Orientierungen einsteigt, gerät allzu leicht ins deduktive Anwenden, und wer beim Urteilen beginnt verliert allzu leicht die übersituativen Strukturen und Orientierungen aus dem Blick.

3 Zur Diskussion dieses Begriffs und der Herkunftsbestimmung anderer Begriffe wie „web 2.0“ oder „social web“ vgl. Höhne 2019, 28. 
Sachgemäß scheint es mir, im Bewusstsein dieser Gefahren bei den theologischen Orientierungen anzusetzen. So werden die evaluativen Voraussetzungen transparent, die implizit nicht nur die Kontextbeschreibung prägen, sondern schon das ethische Agenda-Setting bestimmen, weil vor ihrem Hintergrund erst zum Problem werden kann, was alltagspraktisch vielleicht selbstverständlich scheint, Facebook zu nutzen beispielsweise. Außerdem bewahrt dieser Ansatzpunkt die ethische Reflexion hoffentlich davor, fasziniert oder panisch gebannt auf jede einzelne neue technische Entwicklung zu starren, wie das Säugetier auf die sich erhebende Kobra.

Soll die Grundlegung einer Ethik der Social Media eine theologische sein, stellt sich die Eingangsfrage ein zweites Mal: Wo in der Theologie - bei welchem locus - setzt diese ethische Reflexion ihr Material ordnend und gewichtend an? Plausibel und einschlägig ist der Einstieg über die (theologische) Anthropologie, die - so Alexander Filipović - die Möglichkeit biete, „Sachebene und Sinnebene miteinander zu verknüpfen“ und so Normativität und Empiriebezug zu vermitteln (vgl. Filipović 2012, 25). Die theologische Anthropologie wurde als Ausgangspunkt einer Medienethik über den Begriff der Gottebenbildlichkeit schöpfungstheologisch (Andrea König), trinitätstheologisch (Elmar Kos) oder offenbarungstheologisch und trinitätstheologisch (Christina Ernst) verankert. ${ }^{4}$ Werner Thiede beginnt seine Reflexionen zum digitalen Wandel bzw. zum „Technikwahn“ implizit hamartiologisch, wenn er digitale Vernetzungsversuche als „Turmbau zu Babel" beschreibt: als menschlichen Versuch, wie Gott sein zu wollen. ${ }^{5}$ Und Johanna Haberer steigt - zumindest in einem Abschnitt ihrer „Digitale[n] Theologie" - pneumatologisch-amtstheologisch ein, wenn sie in der allen „Menschen gleichermaßen“ zuteilwerdenden Geistbegabung die „Idee einer

4 Vgl. dazu und zur Identifikation von Andrea Königs Ebenbildlichkeitsverständnis als schöpfungstheologisch und Elmar Kos' als trinitätstheologisch: Costanza und Ernst 2012, 13. Zu König vgl. auch König 2006. Ernst selbst setzt mit Barth bei der menschlich unverfügbaren Dialektik von Sichtbarkeit und Entzogenheit in Gottes Selbstoffenbarung an und versteht von daher dann mit Dalferth und Jüngel Gottebenbildlichkeit trinitätstheologisch (vgl. Ernst 2012, 43-46).

5 Thiede 2015, 7, 2015, 18, 2015, 21-22. In der weiteren Darstellung kippt Thiede zeitweilig ins Apokalyptische: „Was die Urgeschichte prototypisch darstellt, kehrt in weltweitem Maßstab heute endgeschichtlich wieder. Könnte nicht das realutopische Projekt der digitalen Allvernetzung die biblische angesagte Apokalypse befördern?" (Thiede 2015, 19). Das ist insofern konsequent als die Beschreibung der Gegenwart in hamartiologischen Kategorien nach dem erlösenden und versöhnenden Gott fragen lassen muss und nicht nach der ethischen Reflexion menschlichen Verhaltens. 
hierarchiefreien Kommunikation" als Orientierung wachgehalten sieht (vgl. Haberer 2015, 17-18).

In Tradition von vor allem Dietrich Bonhoeffer und Jürgen Moltmann stehend, plädiere ich dafür, die Grundlegung bei eschatologischen Erwägungen zur Wirklichkeit Gottes ansetzen zu lassen. Sofern es dabei um menschliche Hoffnungen geht, manifestieren sich in ihnen ohnehin ethisch leitende Vorstellungen vom guten Leben. Die gemeinte eschatologische Wirklichkeit Gottes ist dabei dem Menschen unverfügbar - und zwar sowohl noetisch, dem erkennenden Menschen, als auch praktisch, dem Handelnden (vgl. Meireis 2008, 259-61): Was Reich Gottes ist, lässt sich weder definieren noch machen. Gleichzeitig orientiert und inspiriert die glaubende Hoffnung auf diese Wirklichkeit menschliches Verhalten, Handeln und Kritik, indem Hoffnung Hoffende in die Hoffnungsrichtung orientiert. ${ }^{6}$ Die eschatologische Grundlegung setzt einen Kontrapunkt zur Orientierung an Dystopien und lässt nach Visionen guten Lebens fragen.

Die Gründe für dieses Plädoyer liegen in den Grundorientierungen, die sich formaliter aus dieser Perspektive ergeben und die ich ähnlich in Anlehnung an Moltmann erarbeitet und anderorts vorgestellt habe (vgl. Höhne 2015, 118-20, 2017a):

Erstens: Integrative Perspektive. Eschatologische Hoffnung zielt auf das Ganze der Wirklichkeit, wird also Engführungen etwa auf einen nur individualethischen Schwerpunkt, zu dem eine primär anthropologische Grundlegung genauso wie eine praxistheoretische Perspektivierung tendiert, nicht aus sich entlassen, ohne diese nicht gleichzeitig als Engführung zu markieren. Ethische Orientierungen für individuelles Handeln und soziale Strukturen im Social Web sind dann gleichermaßen zu betrachten und aufeinander zu beziehen.

Zweitens: Realistische Offenheit. Eschatologische Hoffnung zielt auf die Wirklichkeit Gottes, die schon und noch nicht ist. Damit schafft die Hoffnungsperspektive einen Sinn für das Mögliche und das Fehlende: für Letzteres, weil gerade die Hoffnung ahnen lässt, was zur Erfüllung fehlt,

6 Diesen Gedanken habe ich in Auseinandersetzung mit Jürgen Moltmann angedeutet: Höhne 2015, 59-66. Vgl. auch für das Folgende die Kapitel zu Moltmann in dieser Monographie. Vgl. ähnlich und ebenfalls unter Verwendung des Inspirationsbegriffs: „Gilt im christlichen Kontext das Reich Gottes als höchstes Gutes - im Sinne des Inbegriffs der von Gott aufgerichteten und insofern unverfügbaren universalen Gottesund Menschengemeinschaft im Zusammenhang einer erlösten Schöpfung -, so kann diese zwar nicht als erreichbares Ziel menschlichen Handelns, aber als Inspirationsund Impulsquelle christlicher Bemühung um das Zusammenstimmen aller Tugenden, Pflichten und Güter in und aus Freiheit gedacht werden" (Meireis 2008, 260). 
für Ersteres, weil gerade die Hoffnung nach dem suchen wird, was Gott jetzt schon ermöglicht (vgl. Moltmann 1966, 14, 266f, 308f, 312.). Damit schließt sie hoffnungslose Perspektiven auf die Gegenwart, wie sie etwa ein hamartiologischer Ausgangspunkt nahelegt, genauso aus wie enthusiastische, die die erlittene Unerlöstheit in der Welt übersehen machen. Damit schließt sie auch solche Theorien und Praktiken aus, die nicht mit der Veränderlichkeit von Menschen und sozialen Zusammenhängen rechnen, sondern auf nicht-dynamische Menschen-, Gesellschafts- und Weltbilder festlegen, wohin schöpfungstheologische Reflexionen tendieren können. Was Moltmann $(1966,312)$ über die Welt schrieb, lässt sich auch auf das soziale Netz als Teil dieser Welt übertragen:

„Diese Welt ist nicht der Himmel der Selbstverwirklichung, wie es im Idealismus hieß. Diese Welt ist nicht die Hölle der Selbstentfremdung, wie es in der romantischen und in der existentialistischen Belletristik heißt. Die Welt ist noch nicht fertig, sondern wird als in Geschichte befindlich begriffen. Sie ist darum die Welt des Möglichen, in der man der zukünftigen verheißenen Wahrheit, Gerechtigkeit und dem Frieden dienen kann."

Als Teil der in Geschichte befindlichen Welt ist das soziale Netz weder Himmel noch Hölle, sondern Teil einer unfertigen Welt, in der die Möglichkeiten zu relativ mehr Frieden und Gerechtigkeit ergriffen werden können.

Eschatologische Hoffnung lässt sich von Moltmann herkommend christologisch-kreuzestheologisch ${ }^{7}$ qualifizieren: Die erhoffte Wirklichkeit Gottes geht nicht an den Marginalisierten, Machtlosen und den Opfern der Geschichte vorbei, sondern wird gerade in dem um unsertwillen marginalisierten, entmachteten und gekreuzigten Christus im Anfang offenbar. Das schließt alle Hoffnung aus, die nicht als erstes Hoffnung der Schwächsten und Benachteiligten ist.

Soll eine Ethik der Social Media mit diesen Orientierungen arbeiten und wie vorgeschlagen ihre theologische Grundlegung in eschatologischen Erwägungen finden, muss sie sich gerade deshalb gegen folgende Gefahr wappnen: dagegen nämlich, die fundamentale Differenz von Gottes- und Menschenwerk zu übergehen und Menschen letztlich doch mit der Realisierung des Reiches Gottes zu beauftragen oder ihnen ein Scheitern daran

7 Vgl. Moltmann 1972, 10f., 163, 173. Siehe auch: „Das heißt: das kommende Reich Gottes hat keinen anderen Ort auf dieser Erde als das Kreuz auf Golgatha. Nicht in Zukunftsträumen, sondern im Angesicht des Gekreuzigten sieht uns die Zukunft Gottes an“ (Moltmann 1977, 63). 
vorzuwerfen. ${ }^{8}$ Ersteres kippt in die Überforderung des Menschen, letzteres in die weltverneinende Fundamentalkritik.

\section{Orientierungspunkte: Für das Gute in sozialen Medien}

Mindestens implizit arbeiten auch die bei anderen Loci ansetzenden theologischen Grundlegungen mit orientierenden Hoffnungsvisionen, etwa der Hoffnung auf eine „wahre Identität des Menschen - verstanden als ein seiner Bestimmung vollkommen entsprechendes Sein" (Ernst 2012, 46). Sollen Hoffnungsvisionen in den Zusammenhang mit konkreten Orientierungen gebracht werden, muss - um der angesprochenen Gefahr der Differenznivellierung zwischen unverfügbarer Gotteswirklichkeit und menschlichem Handeln vorzubeugen - dieser Zusammenhang differenziert hergestellt werden. Eine solche Differenzierung hat Torsten Meireis' (2008, 256266. Zitat auf S. 259) „Unterscheidung von unverfügbarem, intendiertem und realisiertem Gutem" in von eschatologischer Perspektivierung her naheliegenden güterethischen Pointierung vollzogen: Der Begriff des „unverfügbaren Guten" verweist bei Meireis darauf, wie Gott die Welt im gottgewirkten Glauben inmitten sündenverzerrter Weltwahrnehmung und deshalb doppelt unverfügbar verstehen lässt (vgl. ebd., 259f): „als gute, aber gefallene Schöpfung Gottes [...], deren Erlösung ebenfalls allein von Gott zu erwarten ist“ (ebd.) und die auf das Reich Gottes „als höchstes Gutes“ ausgerichtet ist (ebd., 260). Artikuliert werde dieses höchste Gut besonders in Bildern, Metaphern und Geschichten, weil diese in ihrer Offenheit gerade die Unverfügbarkeit markieren (vgl. ebd., 264). Als allein von Gott zu Erwartendes, könne dieses Gut „nicht als erreichbares Ziel menschlichen Handelns, aber als Inspirations- und Impulsquelle christlicher Bemühungen um das Zusammenstimmen aller Tugenden, Pflichten und Güter in und aus Freiheit gedacht werden“ (ebd., 260). Damit ist die Differenz zwischen Gottes „Verheißung“ und menschlicher „Verwirklichung“ festgehalten (vgl. Meireis 2008, 261). Der Begriff des „intendierten Gutes“ verweist auf die vom „unverfügbaren Guten“ inspirierten menschlichen Aktivitäten in Bezug auf das unter den begrenzenden Bedingungen der Sünde erstrebte, irdische Gute (vgl. ebd.). Das dritte, das „realisierte Gute“ beinhalte unter den Bedingungen der Sünde auch immer das Böse, bleibt also zweideutig (vgl. ebd., 262).

8 Vgl. zu dieser Gefahr bei Moltmann: Tödt 1967, 198. 
Für eine Ethik der Social Media können besonders diejenigen Hoffnungsbilder impulsgebend sein, die sich auf menschliches Miteinander im Kulturkontext beziehen: Bilder des himmlischen Jerusalem (Offb 21, 1027) oder des harmonischen menschlichen Einstimmens in den innertrinitarischen Chor. ${ }^{9}$ Impulsgebend kann auch das seltener verwendete aber von Markus Mühling $(2007,309)$ ausführlicher besprochene Bild der „eschatische[n] Weinlaube" sein:

„Sie werden ihre Schwerter zu Pflugscharen machen und ihre Spieße zu Sicheln. Es wird kein Volk wider das andere das Schwert erheben, und sie werden hinfort nichtmehr lernen, Krieg zu führen. Ein jeder wird unter seinem Weinstock und Feigenbaum wohnen, und niemand wird sie schrecken. Denn der Mund des HERRN Zebaoth hat's geredet." $(\mathrm{Mi} 4,3 \mathrm{a}-4)^{10}$

Mühling deutet dies als Bild für „umfassendes Heil“, das die Sozialgestalt miteinbezieht, die als zwanglos friedlich und gerecht imaginiert wird, und das im Wein das Geschütztsein des Menschen und sein Eingeladensein zum Gespräch symbolisiert (Mühling 2007, 309). Das derartig imaginierte Gottesreich sieht Mühling neutestamentlich in Jesu „Mahlgemeinschaften mit Sündern" anbrechen (vgl. ebd., 310).

Eine Ausdeutung dieser Bilder ist schon als Reflexion auf das intendierte Gute zu verstehen: In diesen Bilder vermittelt sich materialiter die Vorstellungen von einem guten Leben, in dem jede sich frei, ohne Schrecken (so Micha) entfalten kann, das friedlich ist, weil das Kriegshandwerk verlernt ist, in dem jeder teilhat an Gespräch und gemeinsamen Lebensgenuss, und das deshalb gerecht ist. Formaliter impliziert die Hoffnungsgrundierung, was sich ich in Anlehnung an Moltmanns Arbeit bereits explizit gemacht habe (vgl. Höhne 2015, 188-120):

- die Orientierung an Veränderungsmöglichkeiten in Opposition etwa zu endgültig den Einzelnen festlegenden Menschenbildern,

- die Orientierung an möglichst umfassenden Perspektivierungen in Opposition zur individual- oder sozialethischen Engführung und

- die Orientierung an der vorrangigen Option der Benachteiligten.

9 Für beides, jenes biblische Bild und dieses dort auf Robert W. Jenson zurückgeführte der Musik vgl. Mühling 2007, 307, 315. Mühling ist auch der auf den Kulturkontext im ersten Bild verweist.

10 Luther-Übersetzung, Revision von 2017. In anderer Übersetzung oder Revision zitiert bei Mühling 2007, 309. 
In Bezug auf die Ebene des realisierten Guten werden diese Orientierungen konkreter. Auf dieser Ebene geht es um die „biologischen und sozialen Voraussetzungen“ (Meireis 2008, 262) der Realisierung des Guten - und damit auch um Social Media: Die digitalen Techniken haben nicht nur neue Kommunikationsmöglichkeiten und -medien induziert, sondern wie unter anderem Johanna Haberer festgestellt hat - „einen neuen Lebensraum“ (Haberer 2015, 14) geschaffen, beziehungsweise vorsichtiger und genauer: neue Lebenswelten. In ethischer Perspektive sind es auch digitale Lebenswelten, in denen das irdisch Gute erstrebt wird und zweideutig realisiert ist. Was also kennzeichnet die durch Social Media konstituierten Lebenswelten?

Im Zuge der Digitalisierung sind technische Potenziale entstanden, von denen sich einige mit Neuberger so beschreiben lassen (Vgl. Neuberger 2009, 22f.): Die klassischen Massenmedien ermöglichten Kommunikation von wenigen Sendern zu vielen Empfängern („one-to-many“). „Das Internet integriert Typen von Kommunikation, die durch die Teilnehmerzahl (,one-to-one',,one-to-many' und ,many-to-many'), die Zugänglichkeit von Mitteilungen (öffentlich und privat) sowie die Kommunikationsrichtung (ein- und zweiseitig) bestimmt sind“ (ebd., 23). Netztechnik ermöglicht zudem Mulitmedialität und hat das Potenzial zur Dezentralisierung (ebd., 23-24).

Spezifische Aktualisierungen dieses Potenzials benennen Begriffe wie „web 2.0“ und „social media“ (Neuberger 2009, 22, 30). Mittlerweile einschlägig hat Tim O’Reilly diese Merkmale beschrieben: ${ }^{11}$ Die Kommunikationsmöglichkeiten, die er als „web 2.0“ benennt, unterscheiden sich vom Internet vor dem Platzen der dot-com-Blase unter anderem darin, dass sie das „Netz als Plattform" 12 vorstellen, zu der Nutzer*innen eigene Inhalte beisteuern oder vorhandene Inhalte bearbeiten können, auf der Software als bezahlter und bearbeitbarer Service zur Verfügung steht (und nicht mehr als Softwarepaket gekauft wird), in der Daten durch Algorithmen und die zusammenwirkende Mitarbeit der User*innen dezentral aufbereitet, miteinander vernetzt, geordnet, personalisiert zur Verfügung gestellt werden, etwa: „folksonomy“ (vgl. O’Reilly 2005). Neuberger fasst die Prinzipien des so

11 Vgl. auch für das Folgende O’Reilly 2005. O’Reilly's Beschreibung des „web 2.0“ rezipieren etwa auch Neuberger 2009, 30; Schmidt 2011, 13; Costanza und Ernst 2012, 9. Schmidt hat den Begriff „web 2.0“ „aus wissenschaftlicher Sicht“ zurecht dafür kritisiert, einen Versionssprung zu insinuieren, der mittels des Begriffs beschworen würde, und spricht selbst von „Social Web“ (Schmidt 2011, 14, 24; Costanza und Ernst 2012, 9-10).

12 O’Reilly 2005, meine Übersetzung. 
vorgestellten Kommunikationsraumes mit den Stichworten „Dezentralität“, „Rollenwechsel, Partizipation und Gleichheit“, also auch: Interaktivität, sowie „Vernetzung“ zusammen (vgl. Neuberger 2009, 30; vgl. Höhne 2019, 28). Konkret bezeichnet „Social Web“ damit Anwendungen „wie Soziale Netzwerke [i. e. Facebook, LinkedIn, Xing, StudiVZ, FH], Wikis, OnlineSpiele, Musik- und Filmportale“ (vgl. Costanza und Ernst 2012, 10; Schmidt 2011, 25-30).

Dass damit tatsächlich neue Lebenswelten entstanden sind und das soziale Leben nicht nur auf Marktplätzen und in Kneipen stattfinden, sondern auch, gleichzeitig und stattdessen in den sozialen Netzwerken, führt Jan Schmidts (2011) einschlägige Untersuchung zum „neue[n] Netz“ in praxissoziologischer Perspektivierung deutlich vor Augen: Dazu beschreibt er für das soziale Leben grundsätzlich wichtige „Handlungskomponenten“ der Praktiken, in denen Menschen das Social Web nutzen: Identitätsmanagement, Beziehungsmanagement und Informationsmanagement; Menschen stellten sich online selbst dar, knüpften und unterhielten Kontakte und versorgten sich mit Informationen über ihre Welt (Schmidt 2011, 73). Bei all dem seien sie nicht nur Rezipienten, sondern „aktive Nutzer“ (ebd., 75), die selbst Content erstellen.

Die Vision intendierten, guten Lebens lässt sich vor diesem Hintergrund konkreter fassen. In potentiell partizipativen, dezentralisierten und interaktiven Kommunikationsstrukturen digitaler Lebenswelten, in denen Menschen Identitäts-, Beziehungs- und Informationsmanagement betrieben, erscheint dann Folgendes als konkret erstrebenswert:

- Freie Entfaltung jeder Einzelperson im Schutz vor Gewalt und festnagelnden Menschenbildern.

- Freie Partizipation aller an den interaktiven Möglichkeiten digitaler Lebenswelten.

- Freie Solidarität in der Gestaltung geteilter digitaler Lebenswelten.

Aus den formalen Orientierungen ergibt sich, das Streben danach individual- und sozialethisch zu pointieren, die Gegenwart offen zu halten für Entwicklungen auf diese Ziele hin und primär nach der Realisierung dessen für die am meisten Benachteiligten zu fragen.

\section{Bezugspunkte: Für „gestufte Verantwortung“}

Wer ist dafür verantwortlich? Gerade weil die Kommunikationsmöglichkeiten im Social Web jeden potentiell zum Produser (vgl. Bruns 2009, 117) machen, rückt für eine Ethik der Social Media diese Frage besonders in 
den Vordergrund. Hatte sich die medienethische Debatte lange auf die journalistische Arbeit konzentriert (was plausibel war, solange Journalist*innen Gatekeeper und vorrangige Produzent*innen von Inhalten waren), um dann die Publikumsethik zu entdecken, versuchen neuere Ansätze die Reflexion unterschiedlicher Akteursgruppen und Bereiche zu integrieren: ${ }^{13}$ So geht Rüdiger Funiok $(2011,14)$ von einem „integrativen Konzept von Medienethik" aus und spricht - wohl in Anlehnung auch an R. Spaemann von „gestufte[r] Verantwortung im Medienhandeln“: ${ }^{14}$ „Der Appell, im Prozess der Erstellung, Verteilung und Nutzung von Medienangeboten Verantwortung zu übernehmen, wendet sich an alle, die - in einem gestuften Sinne - Verantwortung tragen" (Funiok 2002). Das erscheint von den entfalteten formalen Orientierungen her als sachgemäß, weil es individualund sozialethische Dimensionen zusammendenkt. Verantwortungsträger für die Lebenswelten des Social Media sind - in Anlehnung an Funioks (vgl. 2011, 14-17) Gliederung: Der Gesetzgeber, der den rechtlichen Rahmen vorgibt, die Programmierer*innen und Architekt*innen von Plattformen, deren aktive und passive Nutzer*innen und die Unternehmen und Körperschaften, die online-Anwendungen zur Verfügung stellen und Geld damit verdienen.

Häufig stehen Verhalten und Verantwortung der Nutzer*innen im Zentrum des Interesses, was gerade wegen deren neuer, über das Rezipieren hinausgehenden Rolle plausibel ist: So hatte etwa das Time-Magazin 2006 die Internetnutzer*innen zur Person des Jahres erklärt. ${ }^{15}$ Und Beck $(2010,143)$ wirft implizit die Frage nach der Verantwortung der Blogger*innen auf, die „ebenso wie diejenigen, die sich dem Journalismus verpflichtet fühlen, zur öffentlichen Kommunikation und Meinungsbildung bei[tragen, FH] “ und deren Texte den „Eindruck erwecken“ können, journalistische zu sein. Undifferenziert die Verantwortung für das gute Leben den Nutzer*innen zuzuschreiben ist dennoch problematisch, weil es ihnen einmal an der Medienkompetenz fehlt, die ausgebildeten, professionellen Journalist*innen unterstellt werden darf, und weil sie zum anderen nicht gleichermaßen aktiv sind. Neben der medienpädagogischen Aufgabe, Medienkompetenz

13 Zur langen Fokussierung auf journalistische Arbeit vgl. Brosda 2010, 258. Vgl. zu ähnlichen Untergliederungen der Medienethik auch Uden 2004, 187; Funiok 2011, 14-17.

14 Funiok 2002. Den Begriff zitiert er andernorts von Spaemann: Funiok 2011, 63.

15 Vgl.: „[... And for seizing the reins of the global media, for founding and framing the new digital democracy, for working for nothing and beating the pros at their own game, TIME's Person of the Year for 2006 is you." (Grossmann 2006, auch zitiert bei: Höhne 2017b). 
zu fördern, kann Verantwortung Nutzer*innen nur nach dem Maße ihrer Kompetenz und Aktivität zugeschrieben werden. Ein anderes Vorgehen stünde in der Gefahr, entweder den Einzelnen zu überfordern oder den Verantwortungsappell ins Diffuse verhallen zu lassen. ${ }^{16}$ Ersteres widerspricht der Orientierung an der Entfaltung des Einzelnen, letzteres der an Partizipation und Solidarität. Nahe liegt also eine Differenzierung der Verantwortungszuschreibung nach einer der zahlreichen Nutzertypologien, die mindestens nach Aktivitätsgrad abstufen. Dafür bietet sich etwa die Empirie basierte Differenzierung von Sabine Haas et al. (2007, 219f.) an, die in dem Koordinatensystem der Dimensionen Gestaltungsgrad und Kommunikationsgrad unterschiedliche Nutzertypen unterschieden haben, beispielsweise: Produzent*innen, Selbstdarstellende, Netzwerker*innen, Informations- oder Unterhaltungssuchende. Mit dem Gestaltungsgrad, also mit der Aktivität in der Nutzung wächst die Verantwortung und die Kompetenzanforderung, mit dem Kommunikationsgrad zwischen individuell und öffentlich (ebd., 220) die Reichweite der Verantwortung: Wer auf Netflix alleine einen Film schaut, trägt nur Verantwortung für sein eigenes Freizeitverhalten (vgl. Funiok 2011, 160f.); wer als Blogger*in mit 10.000 Leser*innen über Babynahrung bloggt, ist für die Folgen seiner bzw. ihrer Publikationen mitverantwortlich.

Gerade weil es bleibend an Medienkompetenz fehlt, weil die technischen Strukturen des Social Web für die meisten Nutzer*innen intransparent sind und weil soziale Prozesse am Werke sind, denen sich der Einzelne als Einzelner kaum entziehen kann, weil durch sozialen Druck Handlungsalternativen etwa zur Mitgliedschaft in einem sozialen Netz kaum bestehen, ${ }^{17}$ greift eine für sich genommene Nutzer*innenethik zu kurz. Sie bedarf der Ergänzung durch eine Unternehmensethik und Programmier*innenethik, die deren Verhalten daraufhin befragt, wie sehr dieses Verhalten dem guten Leben dient, wie weit es also Entfaltung, Partizipation und Solidarität ermöglicht. Die Nutzer*innenethik bedarf vor allem der Ergänzung durch die politisch-ethische Frage nach den gesetzlichen Strukturen und deren Durchsetzung in digitalen Lebenswelten.

16 Vgl. zu diesem Vorbehalt und seinem Vorkommen in der Literatur ausführlich Höhne 2017b und die dort zitierte Literatur.

17 Zur „Macht der Soziabilität“ im Kontext der „Kultur der Digialität“ vgl. Stalder 2016, $160 f$. 


\section{Stichpunkte: Drängende Themen}

Was bedeutet das konkret? Abschließend will ich dies für ein exemplarisches und besonders drängendes Themenfeld einer Ethik der Social Media andeuten: den Datenschutz und -missbrauch im weiteren Sinne. Eine Ethik der Social Media könnte sich natürlich genauso auseinanderzusetzen mit der vor allem wirtschafts- und politikethischen Frage nach der Netzneutralität, den vor allem individual- und politikethischen Fragen nach den Umgangsformen im sozialen Netz oder etwa der medienethischen Grundfrage nach Glaubwürdigkeit und Informationsflut im sozialen Netz. Dabei würden sich die erarbeiteten Orientierungs- und Bezugspunkte als relevant erweisen.

Das Thema Datenschutz und -missbrauch im weiteren Sinne erscheint deshalb als so relevant, weil die Verletzung ,informationeller Selbstbestimmung "18 klammheimlich - weil unmittelbar schadlos - selbstverständlich geworden ist (vgl. Han 2014, 19, 22f; Seele und Zapf 2017, 45). Die seduktive Macht (Han 2014, 27) verführt zur Preisgabe der eigenen Daten im Social Web, die „Macht der Soziabilität“ (Stalder 2016, 160) lässt denen keinen anderen Weg, die partizipieren wollen. Dabei bleibt sowohl die Speicherung als auch Auswertung der preisgegebenen Daten zunächst intransparent, was nicht zuletzt an den für die meisten Nutzer*innen intransparenten Designs liegt (vgl. Han 2014, 22f.).

Ethisch wichtig scheint Datenschutz deshalb, weil die Möglichkeit des Geheimnisses oder des Privaten zur menschlichen Entfaltung dazugehört ${ }^{19}$ und Partizipation und Solidarität erst ermöglicht. Das hat Hannah Arendt (2003, 74f.) gesehen, als sie die Differenzierung von Öffentlichkeit und Privatheit referierte und deren gegenseitige Abhängigkeit betonte. Schon für massenmediale Kommunikation hatte Wolfgang Wunden (1994) Privatheit und Geheimnis als „Grenzen öffentlichen Zeigens" erörtert.

$18 \mathrm{Zu}$ dem Begriff und seinem Verständnis als „normative[m] Konzept“, „Kompetenz“ und „ausgeübte[r] Praxis“ vgl. Schmidt 2011, 124 (Im Original kursiv).

19 So zeigt etwa Schmidt, dass der „Schutz der Privatsphäre“ zusammen mit anderem "grundlegende psychologische Ressourcen dar[stellt], die für die eigene Persönlichkeitsentwicklung und -entfaltung [...] bedeutsam sind" (Schmidt 2011, 117). 
Beim Stichwort Datenschutz und -missbrauch (im nicht immer streng juristischen Sinne) werden staatliche, unternehmerische und publikale Zugriffe auf persönliche Daten thematisiert. ${ }^{20}$ Der publikale Zugriff auf persönliche Daten ist unter digitalen Kommunikationsbedingungen ethisch auf neue und spezifische Weise relevant: Jan Schmidt hat beschreiben, wie sich die von ihm sogenannten „persönlichen Öffentlichkeiten des Social Web" qua Vermittlungstechnik wesentlich von massenmedialen Öffentlichkeiten unterscheiden (vgl. Schmidt 2011, 107-33. Zitat auf S. 118.): Die "Merkmale der kommunikationstechnischen Architektur persönlicher Öffentlichkeiten“ konfrontieren das individuelle Verhalten im Netz „mit unintendierten empirischen Publika in sozialer und/oder zeitlicher Hinsicht" (ebd., 120): Während ich bei einem Brief, einem Zeitungsartikel oder einer Hörfunksendung zumindest bedingt wissen konnte, ein wie großes Publikum darauf Zugriff hat, bleibt dies bei Facebook-Posts oder Blog-Einträgen unsicher: Eltern, Lehrer*innen, Personalverantwortliche können schnell zu einem „unintendierten empirischen Publikum werden“ (vgl. ebd.).

In allen drei Relationen - zum Staat, zu Unternehmen, zu Publika stehen dem aus dem Gut „freie Entfaltung“ abgeleiteten Gut der „informationellen Selbstbestimmung" 21 jeweils nicht nur eindeutig Schlechtes, sondern auch Güter gegenüber: So schwer die demokratische Kontrolle geheimdienstlicher Aktivitäten ist, kann diese Aktivität, insofern sie als Verbrechensprävention fungiert, doch den eigenen Dienst am guten Lebens beanspruchen, wo sie schlicht Leib und Leben etwa vor terroristischen Straftäter*innen schützt. Der unternehmerische Zugriff auf Daten kann für sich in Anschlag bringen, damit nicht nur Geld zu verdienen, sondern auch den Alltag komfortabler zu gestalten. Dies wäre freilich mit mehr Transparenz und Selbstbestimmung auch noch möglich.

Die Frage nach dem Schutz vor „unintendierten empirischen Publika“ (s.o.) ist zunächst ein abgeleitetes Gut: Es dient der freien Persönlichkeitsentfaltung, wenn der Teenager nicht bei jedem Blogeintrag reflektieren muss, welche Konsequenzen es hätte, wenn dieser in 50 Jahren einer

$20 \mathrm{Vgl}$. exemplarisch etwa: https://www.handelsblatt.com/technik/gadgets/wie-staat-und -unternehmen-an-information-kommen-die-datensammler-vom-dienst/3015618.html ?ticket=ST-381487-vURZtgxrTceCkkCSukfH-ap4 [Abruf am 26.04 2019]. Zum hier zugrundeliegenden Verständnis von Überwachung im digitalen Zeitalter vgl. instruktiv Seele und Zapf 2017.

21 Schmidt hat dieses Konzept, das ursprünglich vom Bundesverfassungsgericht geprägt wurde, für das soziale Netz aktualisiert: Schmidt 2011, 124. Vgl. ebd. auch für die Herkunft des Konzepts. Vgl. auch Seele und Zapf 2017, 16. 
breiten Öffentlichkeit zugänglich wird. Diesem Gut steht das Gut der über die gemeinsamen Dinge informierten Öffentlichkeit gegenüber. ${ }^{22}$ Von daher kann es medienethisch als gerechtfertigt erscheinen, aufschlussreiche Jugendvideos eines Spitzenkandidaten einer breiteren Öffentlichkeit zugänglich zu machen.

Die situative Abwägung dieser Güter setzt voraus, dass die Eingangsfrage Thema geworden ist: Wollen wir so leben?

\section{Punkt Punkt Punkt}

Gegen Ende von Marc-Uwe Klings dystopischem Roman hat Peter Arbeitsloser eine Audienz beim Präsidenten, „John of Us“, einem Androiden, gewonnen - und zwar weil sein Anliegen-Post auf der Everybody-Seite des Präsidenten so viel Stimmen anderer Nutzer*innen bekommen hat (vgl. Kling 2017, 364). Peter Arbeitsloser nutzt die Audienz, um Forderungen vorzulesen:

„Alle sollten die Möglichkeiten bekommen, ihre Profile einsehen und korrigieren zu können. Zweitens: Die Arbeitsweise der Algorithmen, die über uns entscheiden, muss transparent gemacht werden, und wir müssen Möglichkeiten bekommen, diese Algorithmen zu beeinflussen. Dazu ist es unbedingt notwendig, dass die Algorithmen ihre Entscheidung begründen! Erst eine Begründung ermöglicht es einem, sinnvoll zu widersprechen!“" (Kling 2017, 368)

In der Dystopie hat Kling aufgezeigt, worauf es eine in der Reflektion von Hoffnungsbildern grundgelegte Ethik der Social Media anlegen kann: die Selbstverständlichkeiten digitaler Lebenswelten in den Diskurs zu überführen, in dem sie hinterfragt und kritisiert werden können - nicht um die digitalen Lebenswelt abzuschaffen, sondern um deren Möglichkeiten im Blick auf ein gutes Leben zu nutzen. Und dieser Diskurs findet selbst in digitalen Lebenswelten statt.

22 Vgl. zur Abwägung von Öffentlichkeit und Persönlichkeitsrecht auch schon Pöttker 1999, 224, 226; Brosda 2010, 262. Vgl. etwa: „Nicht der einzige, aber wohl der wichtigste, jedenfalls ein notorischer Konflikt zwischen allgemeiner Moral und journalistischem Berufsethos ist der zwischen dem professionellen Grundgebot zum Veröffentlichen und dem moralischen Verbot, mit der Persönlichkeitssphäre eines Menschen, über den berichtet wird, auch dessen Würde zu verletzten“ (Pöttker 1999, 226). 


\section{Literaturverzeichnis}

Arendt, Hannah. 2003. Vita activa oder Vom tätigen Leben. Ungekürzte Taschenbuchausgabe, 2. Auflage. Piper 3623. München: Piper.

Beck, Klaus. 2010. „Ethik der Online-Kommunikation.“ In Handbuch Online-Kommunikation, hrsg. von Wolfgang Schweiger und Klaus Beck, 130-55. Wiesbaden: VS Verlag für Sozialwissenschaften.

Brosda, Carsten. 2010. „Journalismus.“ In Handbuch Medienethik, hrsg. von Christian Schicha und Carsten Brosda, 257-77. Wiesbaden: VS Verlag für Sozialwissenschaften.

Bruns, Axel. 2009. „Vom Gatekeeping zum Gatewatching: Modelle der journalistischen Vermittlung im Internet." In Neuberger, Nuernbergk, and Rischke 2009, 107-28.

Costanza, Christina und Christina Ernst. 2012. „Einleitung: Interdisziplinäre Zugänge zu einer Theologie der Social Media." In Costanza and Ernst 2012, 7-16.

Costanza, Christina und Christina Ernst, Hrsg. 2012. Personen im Web 2.0: Kommunikationswissenschaftliche, ethische und anthropologische Zugänge zu einer Theologie der Social Media. Edition Ethik 11. Göttingen: Ruprecht.

Ernst, Christina. 2012. „Sichtbar entzogen: Medienwissenschaftliche und theologische Deutungen von Selbstdarstellungspraktiken auf Facebook.“ In Costanza and Ernst 2012, 32-47.

Filipović, Alexander. 2012. „Anthropologie des Web 2.0? Die Bedeutung eines theologisch-anthropologischen Zugangs für die internetethik." In Costanza and Ernst 2012, 17-31.

Funiok, Rüdiger. 2002. „Medienethik: Der Wertediskurs über Meiden ist unverzichtbar." Zugriff am 1. März 2018. http://www.bpb.de/apuz/2539 $6 /$ medienethik? $\mathrm{p}=$ all.

Funiok, Rüdiger. 2011. Medienethik: Verantwortung in der Mediengesellschaft. 2., durchges. und aktualisierte Aufl. Kon-Texte 8. Stuttgart: Kohlhammer.

Grossmann, Lev. 2006. „You - Yes, You - Are TIME's person of the Year: $n$ 2006, the World Wide Web became a tool for bringing together the small contributions of millions of people and making them matter." Zugriff am 1. Oktober 2018. http://content.time.com/time/magazine/art icle/0,9171,1570810,00.html.

Haas, Sabine, Thilo Trump, Maria Gerhards und Walter Klingler. 2007. „Web 2.0: Nutzung und Tutzertypen." Media Perspektiven (4): 215-222. 
Haberer, Johanna. 2015. Digitale Theologie: Gott und die Medienrevolution der Gegenwart. München: Kösel.

Han, Byung-Chul. 2014. Psychopolitik: Neoliberalismus und die neuen Machttechniken. Frankfurt am Main.

Höhne, Florian. 2015. Einer und alle: Personalisierung in den Medien als Herausforderung für eine Öffentliche Theologie der Kirche. Öffentliche Theologie 32. Leipzig: Evang. Verl.-Anst. Teilw. zugl. Erlangen-Nürnberg, Univ., Diss., 2014 u.d.T. Personalisierung in den Medien als Herausforderung für eine evangelische Öffentliche Theologie der Kirche. Höhne, Florian. 2017a. „Prophetenrufe und Königsbilder: Anregung zu einer Ethik ästhetischer Formen im Horizont Öffentlicher Theologie." In Öffentliche Theologie zwischen Klang und Sprache: Hymnen als eine Verkörperungsform von Religion, hrsg. von Thomas Wabel, Florian Höhne und Torben Stamer, 41-68. Öffentliche Theologie 34. Leipzig: Evangelische Verlagsanstalt.

Höhne, Florian. 2017b. "Who Is Responsible for What I Do on Facebook to Democracy? A Public-Theological Reflextion on the "Responsibility" of Media-Users for Democratic Culture." In Religion and Democracy: Studies in Public Theology, hrsg. von Torsten Meireis und Rolf Schieder. 1. edition, 121-41. Ethik und Gesellschaft volume 3. Baden-Baden, Germany: Nomos.

Höhne, Florian. 2019. „Darf ich vorstellen: Digitalisierung. Anmerkungen zu Narrativen und Imaginationen digitaler Kulturpraktiken in theologisch-ethischer Perspektive." In Digitaler Strukturwandel der Öffentlichkeit. Interdisziplinäre Perspektiven auf politische Partizipation im Wandel, hrsg. von Jonas Bedford-Strohm, Florian Höhne und Julian ZeyherQuattlender, 25-46. Kommunikations- und Meidnethik 10. BadenBaden, Germany: Nomos.

Kemper, Peter, Alf Mentzer und Julika Tillmanns, Hrsg. 2012. Wirklichkeit 2.0: Medienkultur im digitalen Zeitalter. Reclam-Taschenbuch 20266. Stuttgart: Reclam.

Kling, Marc-Uwe. 2017. Qualityland. 3. Aufl. Berlin: Ullstein.

König, Andrea. 2006. Medienethik aus theologischer Perspektive: Medien und Protestantismus - Chancen, Risiken, Herausforderungen und Handlungskonzepte. Marburg: Tectum-Verl. Univ., Diss.-Regensburg, 2005.

Meireis, Torsten. 2008. Tätigkeit und Erfüllung: Protestantische Ethik im Umbruch der Arbeitsgesellschaft. Tübingen: Mohr Siebeck. Zugl. Münster (Westfalen), Univ., Habil.-Schr., 2007. 
Moltmann, Jürgen. 1966. Theologie der Hoffnung: Untersuchungen zur Begründung und $z u$ den Konsequenzen einer christlichen Eschatologie. 6. Aufl. Beiträge zur evangelischen Theologie 38. München: Chr. Kaiser Verlag.

Moltmann, Jürgen. 1972. Der gekreuzigte Gott: Das Kreuz Christi als Grund und Kritik christlicher Theologie. München: Kaiser.

Moltmann, Jürgen. 1977. „Hoffnung und Entwicklung.“ In Zukunft der Schöpfung: Gesammelte Aufsätze, hrsg. von Jürgen Moltmann, 59-67. München: Kaiser.

Mühling, Markus. 2007. Grundinformation Eschatologie: Systematische Theologie aus der Perspektive der Hoffnung. Göttingen: Vandenhoeck \& Ruprecht.

Neuberger, Christoph. 2009. „Internet, Journalismus und Öffentlichkeit: Analyse des Medienumbruchs." In Neuberger, Nuernbergk, and Rischke 2009, 19-105.

Neuberger, Christoph, Christian Nuernbergk und Melanie Rischke, Hrsg. 2009. Journalismus im Internet: Profession - Partizipation - Technisierung. Wiesbaden: VS Verlag für Sozialwissenschaften.

O'Reilly, Tim. 2005. "What Is Web 2.0: Design Patterns and Business Models for the Next Generation of Software." Zugriff am 1. März 2018. http://www.oreilly.com/pub/a/web2/archive/what-is-web-20.html.

Pariser, Eli. 2012. „Wie wir im Internet entmündigt werden.“ In Wirklichkeit 2.0: Medienkultur im digitalen Zeitalter, hrsg. von Peter Kemper, Alf Mentzer und Julika Tillmanns, 58-69. Reclam-Taschenbuch 20266. Stuttgart: Reclam.

Pöttker, Horst. 1999. „Öffentlichkeit als gesellschaftlicher Auftrag: Zum Verhältnis von Berufsethos und universaler Moral im Journalismus." In Medienethik - die Frage der Verantwortung, hrsg. von Rüdiger Funiok, Udo F. Schmälzle und Christoph H. Werth, 215-32. Bonn.

Reuter, Hans-Richard. 2015. „Grundlagen und Methoden der Ethik." In Handbuch der evangelischen Ethik, hrsg. von Wolfgang Huber, Torsten Meireis und Hans-Richard Reuter, 9-123. München: C.H. Beck.

Schmidt, Jan-Hinrik. 2011. Das neue Netz: Merkmale, Praktiken und Folgen des Web 2.0. 2., überarb. Aufl. Kommunikationswissenschaft. Konstanz: UVK.

Seele, Peter und Chr. Lucas Zapf. 2017. Die Rückseite der Cloud: Eine Theorie des Privaten ohne Geheimnis. Berlin, Heidelberg: Springer Berlin Heidelberg; Imprint: Springer. 
Stalder, Felix. 2016. Kultur der Digitalität. Edition Suhrkamp 2679. Berlin: Suhrkamp.

Thiede, Werner. 2015. Digitaler Turmbau zu Babel: Der Technikwahn und seine Folgen. München: Oekom-Verl.

Tödt, Heinz Eduard. 1967. „Aus einem Brief an Jürgen Moltmann.“ In Diskussionen über die "Theologie der Hoffnung" von Jürgen Moltmann, hrsg. von Wolf-Dieter Marsch, 197-200. München: Chr. Kaiser Verlag. Tödt, Heinz Eduard. 1988. Perspektiven theologischer Ethik. München: Kaiser.

Uden, Ronald. 2004. Kirche in der Medienwelt: Anstöße der Kommunikationswissenschaft zur praktischen Wahrnehmung der Massenmedien in Theologie und Kirche. Studien zur Christlichen Publizistik 10. Erlangen: CPV Christliche-Publizistik-Verl. Univ., Habil.-Schr.-Erlangen-Nürnberg, 2002.

Wunden, Wolfgang. 1994. „Grenzen öffentlichen Zeigens: Privatheit als Element einer Kultur der Öffentlichkeit." In Öffentlichkeit und Kommunikationskultur, hrsg. von Wolfgang Wunden, 165-79. Beiträge zur Medienethik 2. Hamburg, Stuttgart, Frankfurt am Main: Steinkopf [u.a.]. Zugriff am 26. April 2019. 